\title{
Treinta años de la Casa de las Américas
}

Roberto Fernández Retamar

Es difícil ser juez y parte, y en tal situación me encuentro ahora. Casi desde su nacimiento ofrecí conferencias en la Casa de las Americas, y luego fui miembro del jurado de su Premio Literario y colaborador de la que era entonces su única revista. Desde 1965 comence a dirigir esa revista, y desde 1986 a presidir la institución. Eso quiere decir que estoy profundamente identificado con ella. En consecuencia, puedo mencionar sus tareas, pero no estoy autorizado (ya que carezco de objetividad) para calificarlas. Permítaseme pues dar más de una vez la palabra a otros en las páginas que siguen.

Siempre he pensado que la creación por la Revolución Cubana, a menos de cuatro meses de su triunfo en enero de 1959, de la Casa de las Américas, es uno de los hechos que revelaron tempranamente el carácter no local sino latinoamericano y caribeño de la Revolución. Como es natural, no podían estar previstas en todos sus detalles las que iban a ser realizaciones concretas de la institución. En esto, como en tantas cosas, "se hace camino al andar", según el clásico verso machadiano. Pero que la intención primordial fue aquella, no deja lugar a dudas. Como tampoco deja lugar a dudas el que la Casa haya nacido bajo la conducción de la legendaria heroína del Moncada y de la Sierra Maestra que fue Haydee Santamaría. Este último hecho mostro con claridad la importancia concedida desde el primer instante a la institución entonces naciente.

El criterio que alentaba la decisión de crear la Casa de las Américas era el de la necesaria unidad de lo que Martí llamo Nuestra América: la América bolivariana, martiana, sandinista. Se sabe que no hay acontecimiento político y social de veras trascendente en nuestros países que no haya hecho suyo el proyecto de unidad continental: el proyecto cuya primera figura solar fue Simon Bolívar, y que en nuestros dias encontro un heraldo excepcional en la figura de Ernesto Che Guevara, quien nació en la Argentina, fue uno de los pilares de la Revolución Cubana y murió en Bolivia al frente de lo que estaba en vias de ser un nuevo ejercito bolivariano. Para impulsar la realización de ese proyecto, sobre todo en lo tocante a la cultura artística, surgio la Casa de las Américas. Su objetivo inmediato era (y es) estrechar los vínculos entre los artistas y escritores latinoamericanos y caribeños, y difundir sus obras a lo largo y ancho del Continente, y aún del mundo. Naturalmente, tal propósito tenía claros antecedentes: de Andrés Bello a Juan María Gutierrez, de Francisco Bilbao a José Marti, esos antecedentes jalonan lo mejor de nuestra historia intelectual en el siglo XIX. Y ya en éste, son evidentes los estímulos recibidos de acontecimientos como la Revolución Mexicana de 1910, con tan ricas consecuencias culturales, y la Revolución Guatemalteca que en 1954 fue ahogada en sangre por el imperialismo norteamericano; y de obras como las del dominicano Pedro Henriquez Ureña, cuya sabia hermana Camila fue activa colaboradora de la $\mathrm{Ca}$ sa. Siempre sera motivo de particular orgullo para la Casa el que el Che (entre cuyas experiencias se encontraban sus días guatemaltecos y mexicanos) la visitara en varias ocasiones, e incluso afirmara en un discurso dicho en Punta del Este, Uruguay, en 1961, que la Casa se proponía la "exaltación del patrimonio cultural de toda Nuestra América"; y que también la visitara, estudiara en ella, nos aconsejara y colaborara con nosotros Carlos Fonseca. 


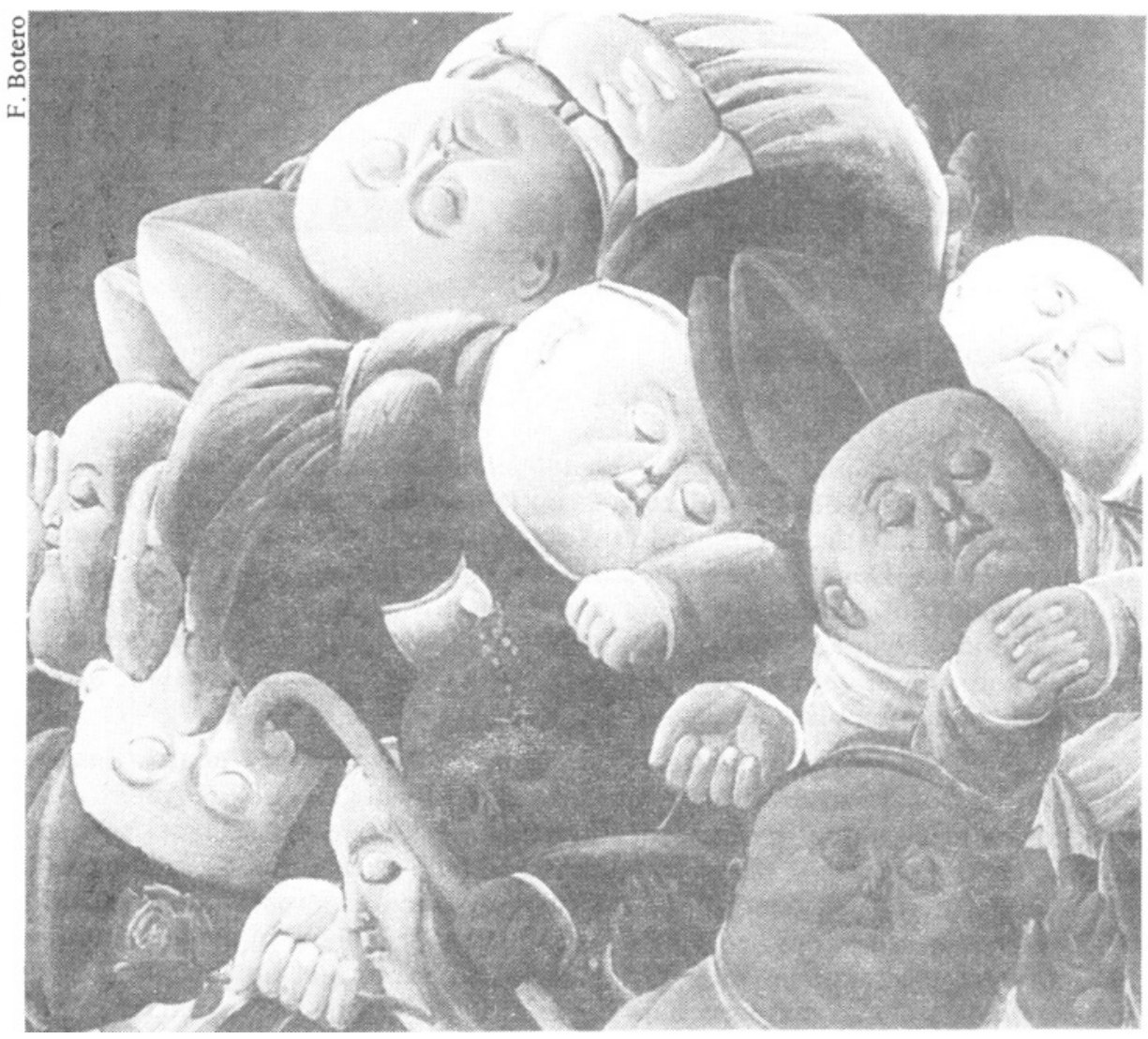

No llevaba mucho tiempo de fundada la Casa cuando todos los gobiernos latinoamericanos, con la noble excepción del de México, fueron rompiendo relaciones diplomáticas con Cuba, por añadidura expulsada de la OEA. En esas condiciones, correspondió a la Casa ser uno de los vehículos que impidieron el total aislamiento latinoamericano de la Cuba revolucionaria. Incuestionablemente, el mayor mérito de este hecho recae en los cuantiosísimos escritores y artistas de nuestros países que contra viento y marea persistieron en sus relaciones con la Casa de las Américas, que era como decir con la Revolución Cubana y mejor aún con la Revolución Latinoamericana. Esas relaciones implicaron en no pocos casos dificultades de diversa naturaleza para aquellos escritores $\mathrm{y}$ artistas que se mantuvieron fieles a las mejores tradiciones y las esperanzas mejores de Nuestra América. Desde hoy, les ratificamos nuestra gratitud y nuestra admiración a esos compañeros que vemos representados, para sólo nombrar a unos pocos desaparecidos, en Ezequiel Martínez Estrada, Manuel Galich y Roque Dalton, entre quienes trabajaron en nuestros locales; y entre quienes también nos acompañaron lealmente, en Efraín Huerta, Jorge Zalamea, Sebastián Salazar Bondy, Benjamín Carrión, Manuel Rojas, Víctor Jara, Paco Urondo, Rodolfo Walsh, Haroldo Conti, Julio Cortázar, Mario Arregui, César Fernández Moreno, Angel Rama, Lilia Carrillo, Miguel Otero Silva, César Rengifo, Aquiles Nazoa, Francisco Manrique Cabrera, Juan Aburto, y por supuesto en cubanos como Juan Marinello, Alejo Carpentier, José Lezama Lima, Mirta Aguirre, Onelio Jorge Cardoso, Fayad Jamís, Luis Rogelio Nogueras.

En el Informe del Comité Central del Partido Comunista de Cuba al Primer Congreso de dicho Partido, presentado en 1975 por el compañero Fidel Castro, se dijo lo siguiente: 
"La fundación en 1959 de la Casa de las Américas contribuyó a impedir el aislamiento cultural en los momentos más difíciles del bloqueo, y mediante diversas actividades como publicaciones, concursos, premios, festivales, exposiciones y encuentros de literatura, teatro, plástica y música, ha vinculado a la Revolución Cubana los sectores más progresistas de la intelectualidad latinoamericana y a latinoamericanistas de los países socialistas, manteniendo en el Continente el aliento de la Revolución Cubana. La Casa de las Américas constituye hoy el centro cultural más prestigioso de América Latina."

Aquí se mencionan funciones de varios de los departamentos de la Casa: los de Literatura, Teatro, Artes Plásticas, Música, Editorial. La Casa cuenta también con un Centro de Estudios del Caribe, una Biblioteca especializada en cuestiones del área, y otros departamentos que contribuyen a hacer posible su faena.

Quizás la más conocida de las actividades de la Casa sea el Premio Literario Casa de las Américas, que se convocó por vez primera recién creada la Casa y se otorgó a principios de 1960. Entre los premiados de entonces, uno era un hombre ya consagrado continentalmente: el argentino Ezequiel Martínez Estrada; otro, un escritor inédito hasta la fecha: el cubano José Soler Puig, quien en 1975, con la publicación de El pan dormido, se reafirmaría entre los grandes novelistas americanos. Ese amplio radio, que va desde los reconocidos hasta los noveles, se mantendría como una constante del Premio durante los que van a ser sus primeros treinta años. Durante ese tiempo, los jurados han provenido de los más diversos países de Nuestra América y aún de los Estados Unidos, Europa y Africa. Por otra parte, en los primeros momentos sólo se otorgaban los premios a obras escritas en español en los cinco géneros tradicionales: novela, cuento, poesía, obra de teatro y ensayo. Posteriormente, el horizonte se fue ampliando con nuevos géneros y nuevos idiomas, y en la actualidad se conceden también en testimonio (el nuestro fue probablemente el primer concurso que acogió este género) y literatura para niños, y se conceden igualmente en portugués (para autores brasileños) e inglés y francés (para autores caribeños de estas lenguas). Además, cada cierto tiempo se convoca a premios extráordinarios: ya se han otorgado los correspondientes a Simón Bolívar, José Carlos Mariátegui, Ernesto Che Guevara y La Juventud en Nuestra América. Para 1989 se ha convocado al Premio Extraordinario XXX aniversario de la Revolución Cubana, con motivo de las tres décadas que en la fecha cumplirá la Revolución; y para 1991, al Premio Extraordinario Nuestra América: ese año tendrá un siglo de aparecido el extraordinario ensayo de Martí así llamado.

Además del Premio Literario, la Casa otorga otros en Artes Plásticas (Grabado y Fotografía) y Música (Musicología). Y junto a la revista homónima, que es su vocero, edita también las publicaciones periódicas Conjunto (dedicada al teatro), Música y Anales del Caribe, todas ellas dedicadas a Nuestra América, y coedita, conjuntamente con la Unión de Escritores y Artistas de Cuba, Criterios, revista de teoría literaria, estética y culturología. Pero, como es natural, el grueso de las publicaciones de la Casa recae en los libros que hace nuestra Editorial, la cual cuenta ya con centenares de títulos y las colecciones Premio, Literatura Latinoamericana, La Honda, Cuadernos de la Casa de las Américas, Pensamiento de Nuestra América, Nuestros Países, Valoración Múltiple y otras. A través de esa variada red, la Casa divulga las obras que

Quizás la más conocida de las actividades de la Casa sea el Premio Literario Casa de las Américas, que se convocó por vez primera recién creada

la Casa y se otorgó a principios de 1960. premia, los clásicos y los contemporáneos de nuestra literatura, las obras de nuestros principales pensadores, ensayos sobre nuestra realidad. Además la Casa edita catálogos, afiches, discos: estos últimos unas veces de música, y otras con textos literarios por lo general leídos por sus autores, cuyas voces se conservan en el Archivo de la Palabra de la institución.

La Casa cuenta con dos galerías de arte: la Galería Latinoamericana, que radica en su local principal, y la Galería Haydée Santamaría, que muestra la Colección Permanente de Arte de Nuestra América. Aunque ambas lo hacen, 
corresponde sobre todo a la primera acoger exposiciones temporales tanto de obras de uno o varios autores como de manifestaciones anónimas, mientras la principal tarea de la Galerfa Haydee Santamaría es mostrar una parte apreciable de las muchas obras que a lo largo de estos años nos han sido donadas por sus autores o por personalidades relevantes de los distintos países latinoamericanos y caribeños.

Cada uno de los departamentos organiza, de acuerdo con su naturaleza, conferencias, lecturas, mesas redondas, exposiciones, conciertos, representaciones teatrales o muestras de video, que tienen lugar constantemente. Además, atesora una vasta información sobre las áreas respectivas, y realiza de tiempo en tiempo encuentros de escritores, teatristas, plásticos, músicos o educadores populares. Estas reuniones permitem hacer un balance y proyectar tareas en común. La meta es siempre esa: trabajar concertadamente, dejando de lado lo pequeño que pueda dividirnos y subrayando lo grande que nos une y lo diverso que nos enriquece. En este sentido, las reuniones de mayor envergadura que hemos realizado son los dos Encuentros de Intelectuales por la Soberanía de los Pueblos de Nuestra América, uno en 1981 y otro en 1985, en que a los intelectuales con que solemos trabajar se integraron científicos, periodistas, té6logos, unificados en el rechazo a la prepotencia imperialista y en la defensa de nuestros auténticos valores.

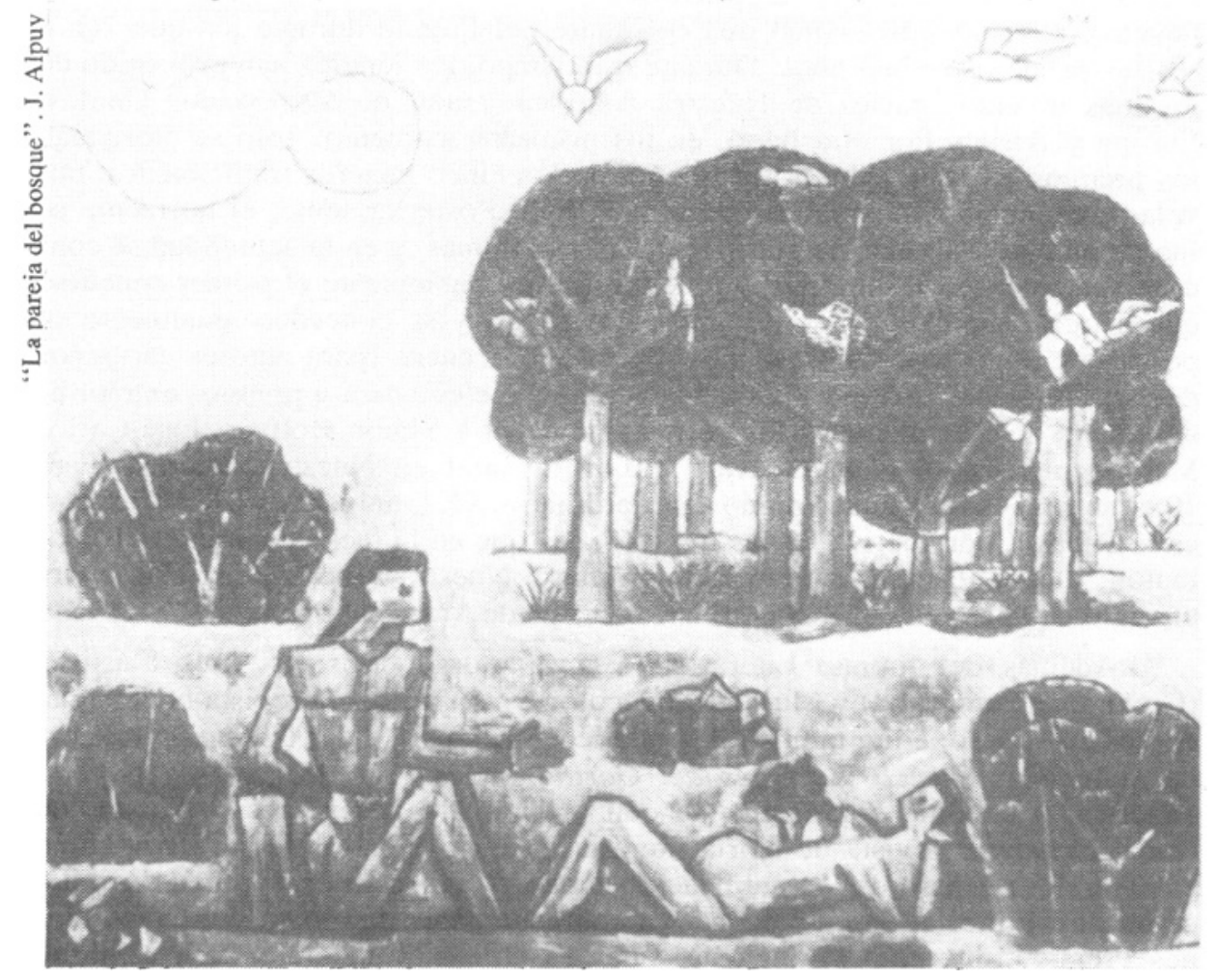

Podria hablar largamente de nuestra Biblioteca (que es también hemeroteca), sin la cual no nos sería posible realizar muchas de nuestras labores; y tambien podría hablar de otras esquinas de nuestra Casa. Pero por mucho que intente describir tales esquinas, y por mucho que mencione sus faenas, poco habre dicho si no logro trasmitir el espíritu de trabajo que anima a la Casa, un espíritu que en lo esencial no se ha modificado desde los años en que una gran dirigente política, Haydee Santamaria, estuvo al frente de la institución, hasta los años más cercanos en que a ese frente han estado un excelente pintor, Mariano Rodriguez, y luego un poeta. Creo que se trata fundamentalmente de un espíritu de servicio con que Haydée marcó a la Casa, y que hace que sus tra- 
bajadores suelan sentir el orgullo de serlo en un centro que tiene là responsabilidad (y la convicción) de ser útil. Sin duda en esto ha sido determinante la inmensa presencia de Haydé, su hechizo personal, su manera inolvidable de vincular la política radical y la sensibilidad humanista, su convicción de que tan trabajadores de la Casa son los que laboran en sus locales como los que lo hacen en otros lugares y países, su necesidad orgánica de justicia y de belleza. Como consecuencia de ello, y junto a su esencial vocación latinoamericanista y caribeña, la Casa ha mantenido una permanente exigencia de verdad y de calidad (no sólo artística), y una renovada voluntad de contemporaneidad. Esto último podrá parecer extraño a quien conozca como la Casa ha luchado y lucha por preservar lo mejor de nuestras tradiciones. Pero en cambio será perfectamente comprensible para quien recuerde las palabras de Stravinsky según las cuales una tradición no es algo muerto, sino una fuerza viva que informa $y$ anima al presente. Precisamente a esa mezcla de defensa de nuestros genuinos valores, exigencia de calidad y afirmación de la contemporaneidad creo que se debe en apreciable medida, el vivo respaldo que Ia mayoría de los intelectuales latinoamericanos y caribeños dan a la Casa de las Américas.

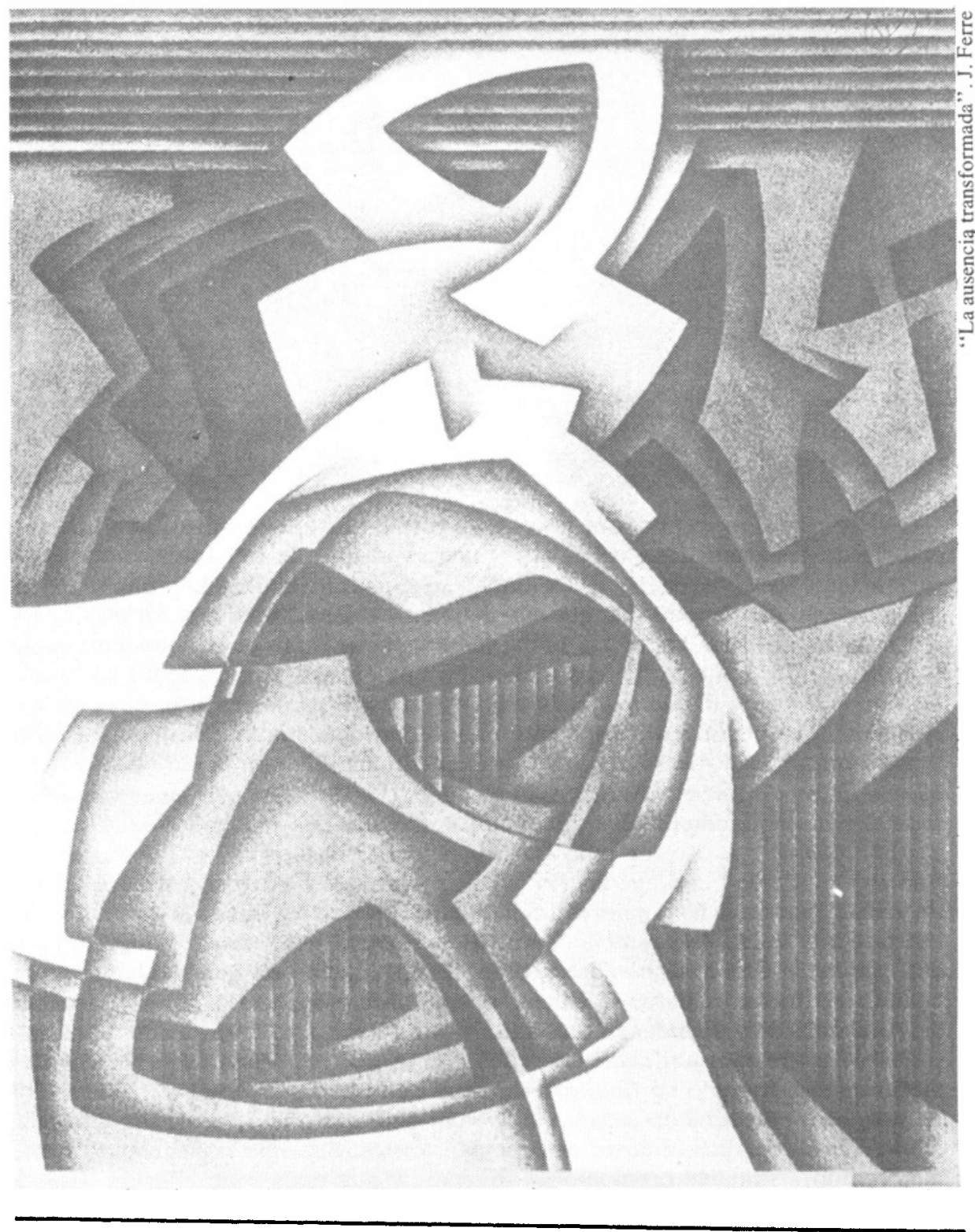




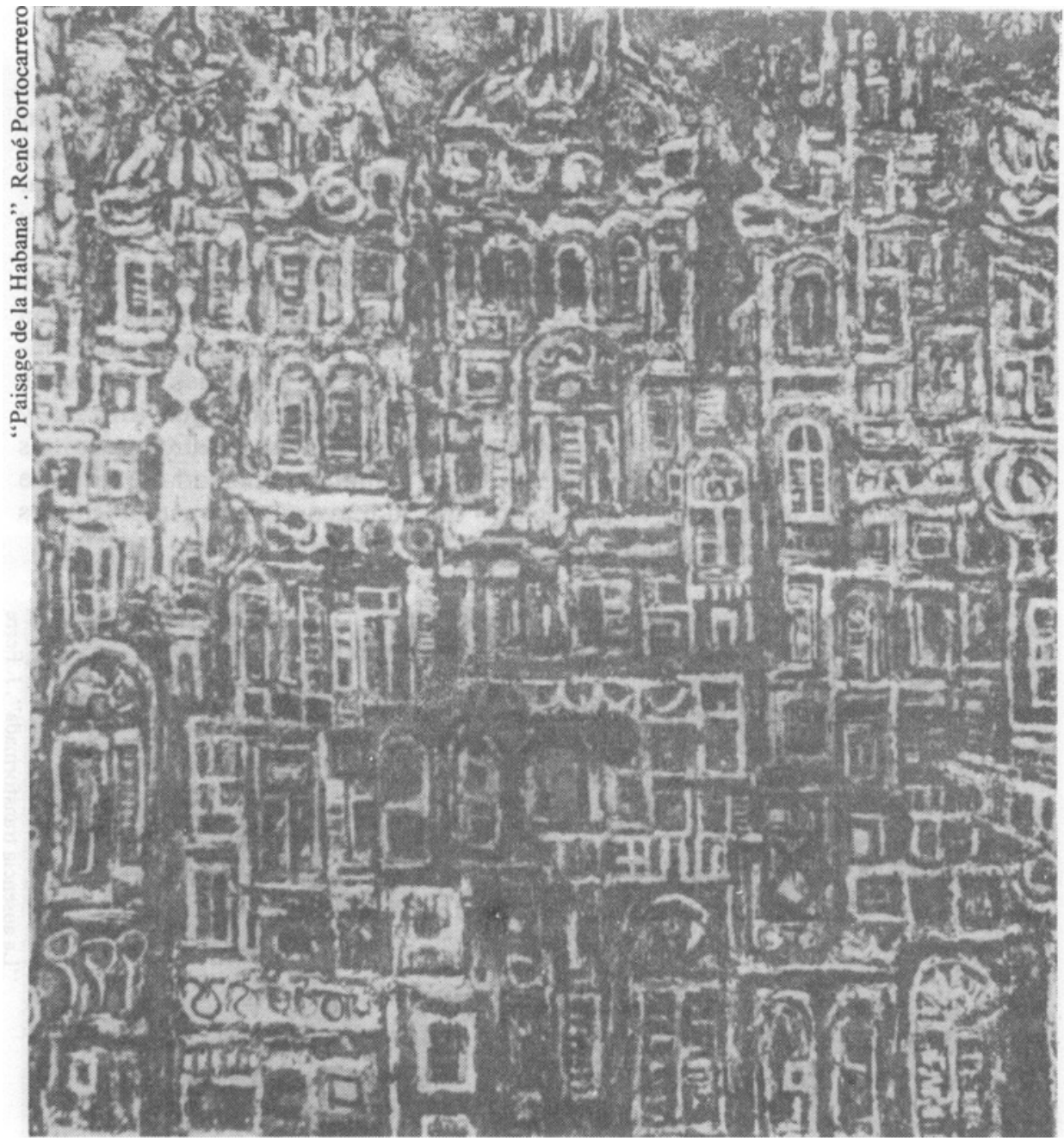

Ese respaldo, está claro, no es a una rígida institución local ni a una suma amorfa de departamentos o labores. Pues es propio de la Casa su voluntad de dinamismo, de coherencia; su voluntad de elaborar, o de relaborar, junto con los muchísimos compañeros que tenemos en nuestra América y en otras partes del mundo, criterios sobre las multiples cuestiones que constantemente hemos ido abordando. No está de más insistir en que entre esas cuestiones no ha estado nunca la torpe tentación de pretender señalarle pautas al arte, cuya rica variedad y cuya búsqueda permanente le son consustanciales. Ni hemos aspirado a una uniformidad ideológica que no sería compatible con la variedad de situaciones que vive nuestra America. Pero tampoco podíamos permanecer neutrales ante el panorama complejo y a menudo dramático que muchos de nuestros pafses han ofrecido $u$ ofrecen aún. La neutralidad, en estos casos, hubiera equivalido a complicidad con el imperio. Y precisamente el honroso rechazo a éste es un rasgo común de los mejores intelectuales latinoamericanos y caribeños. Por eso la Casa de las Américas ha combatido cuantas maniobras el imperio ha urdido en estas décadas peleadoras para desviar hacia una neutralidad culpable, cuando no hacia la defensa abierta de sus intereses, a escritores y artistas a quienes unas veces amenaza o coarta, y otras halaga zalameramente para apartarlos de las responsabilidades de ellos con respecto a sus pueblos. Y si bien es cierto que ha logrado su finalidad en unos cuantos casos, y ya se habla incluso de una nueva derecha en la vida intelectual de nuestro continente, es sobre todo cierto que a aquellos, enormemente mayoritarios, que han permanecido firmes, han venido a sumarse promociones jóvenes, y que unos y otros están llamados 
a configurar una nueva izquierda que imaginativa y valientemente dé respuestas adecuadas a las actuales circunstancias. Así vemos las cosas en la Casa de las Americas. Y colaborar en ello nos parece nuestro deber actual.

Evaluando en 1979 el trabajo cumplido hasta esa fecha por la Casa de las Américas, Mario Benedetti escribi6:

"Desde su creación, la Casa quiso ser un centro de difusión de estudio y de encuentro del arte y las letras latinoamericanas, o sea, una nueva forma de lucha contra la segmentación y el desmembramiento de nuestra cultura, fomentados desde siempre por el imperialismo. Por lo general, los escritores y artistas latinoamericanos y del Caribe sabian más de lo que se producía en Paris, Londres o Nueva York que de lo que se creaba en México, Caracas, La Habana, Lima, Buenos Aires, Kingston o Montevideo. La Casa de las Américas propició el encuentro en Cuba de escritores, pintores, músicos, dramaturgos, cantantes; muchos de nosotros nos conocimos aquí, dialogamos aquí, intercambiamos aquí por vez primera experiencias y opiniones sobre la vida artística de nuestros respectivos países." Cubana,

Y en 1980 añadía Julio Cortázar que en la decisión de la Revolución

"de dar el máximo, de proyectarse más allá de la orbita local como la única manera de encontrarse auténticamente consigo mismo, la labor de la Casa de las Américas asume una significación que ningún elogio podría abarcar, y que sobrepasa largamente su breve vida institucional. (...) En estos últimos años la irradiación cultural de la Casa se ha visto multiplicada por muchas razones, que sólo mencionaré parcialmente. En primer lugar, sus publicaciones y actividades han ocupado un lugar permanente y muy importante en todos los centros de recepción de cultura del mundo, incluso en algunos cuya línea ideologica dista de ser la de Cuba pero que ya no pueden ignorar la calidad y la validez de la producción intelectual y artística que la Casa vehicula y estimula".

Por último, en un coloquio universitario celebrado en el Brasil en octubre de este año, y en el que se rendía homenaje tanto a la Casa de las Americas como a él mismo, el profesor brasileño Antonio Candido recordo el interés suscitado en estas décadas por convocar reuniones de intelectuales latinoamericanos en países metropolitanos, como fue el caso del Congreso realizado, bajo los auspicios del Columbianum, en Génova, en enero de 1965; y añadio:

"En ese momento ya estaba en plena actividad una alternativa nuestra, esto es, una nueva modalidad que consistía en promover de manera sistemática el encuentro de los intelectuales y artistas latinoamericanos en la propia América Latina, sin mediaciones, por más positivas que fuesen, como era la del Columbianum. Me refiero al esfuerzo heroico de una institución justamente homenajeada en este coloquio, la Casa de las Americas, a cuyo actual presidente, Roberto Fernández Retamar, conocí en el Congreso de Génova, hace casi veinticuatro años. En la lucha gigantesca por romper el aislamiento que le fue impuesto, la República de Cuba de-

Ni hemos aspirado a una uniformidad ideologica que no serfa compatible con la variedad de situaciones que vive nuestra América. sarrollo con enormes sacrificios un sistema de encuentros internacionales de todo tipo, que permitiría a los intelectuales y artistas de la América Latina el contacto constante en el terreno del propio sudcontinente, sin necesidad de intermediarios. Vinculado personalmente a la Casa de las Américas por más de una actividad de colaboración, puedo testimoniar sobre la importancia de este esfuerzo que dio nuevo timbre a nuestra convivencia."

Roberto F. Retamar é poeta e crítico literário cubano e diretor da Casa de las Américas (Havana). 